Пантелеев И.А., Плехов О.А., Наймарк О.Б., Евсеев А.В., Паньков И.Л., Асанов В.А. Особенности локализации деформации при растяжении сильвинита // Вестник ПНИПУ. Механика. - 2015. - № 2. - C. 127-138. DOI: 10.15593/perm.mech/2015.2.08

Panteleev I.A., Plekhov O.A., Naimark O.B., Evseev A.V., Pankov I.L., Asanov V.A. Features of strain localization in sylvinite under tension. PNRPU Mechanics Bulletin. 2015. No. 2. Pp. 127-138. DOI: 10.15593/perm.mech/2015.2.08

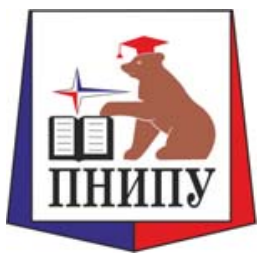

ВЕСТНИК ПНИПУ. МЕХАНИКА

№ 2, 2015

PNRPU MECHANICS BULLETIN

http://vestnik.pstu.ru/mechanics/about/inf/

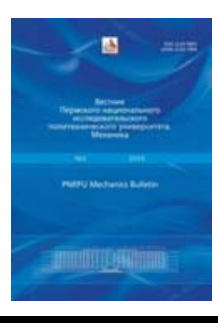

DOI: $10.15593 /$ perm.mech/2015.2.08

УДК 539.42

\title{
ОСОБЕННОСТИ ЛОКАЛИЗАЦИИ ДЕФОРМАЦИИ
}

\section{ПРИ РАСТЯЖЕНИИ СИЛЬВИНИТА}

\author{
И.А. Пантелеев ${ }^{1}$, О.А. Плехов ${ }^{1}$, О.Б. Наймарк ${ }^{1}$, А.В. Евсеев ${ }^{2}$, \\ И.Л. Паньков ${ }^{2}$ В.А. Асанов ${ }^{2}$ \\ ${ }^{1}$ Институт механики сплошных сред УрО РАН, Пермь, Россия \\ ${ }^{2}$ Горный институт УрО РАН, Пермь, Россия
}

O CTATЬE

Получена: 18 марта 2015 г.

Принята: 1 мая 2015 г.

Опубликована: 30 июня 2015 г.

Ключевые слова:

локализация деформации,

акустическая эмиссия,

прямое растяжение,

концентрационный параметр,

метод корреляции цифровых

изображений

\begin{abstract}
АННОТАЦИЯ
Настоящая работа посвящена исследованию пространственно-временной локализации деформации при прямом одноосном растяжении сильвинита с использованием метода корреляции цифровых изображений и метода акустической эмиссии. Для реализации схемы прямого одноосного растяжения использовано специальное реверсивное устройство, в котором бетонируются образцы сильвинита. Последовательно сфотографированные изображения боковой поверхности образцов были использованы для восстановления распределения полей компонент вектора перемещений и тензора деформаций на боковой поверхности образцов сильвинита при одноосном квазистатическом растяжении. В результате анализа полученных данных установлено, что процесс деформирования реализуется в виде двух последовательно следующих форм пространственно-временной локализации: системы эквидистантно расположенных стационарных очагов локализованной деформации и одиночной стационарной диссипативной локализованной структуры. Материал вне полос локализованной деформации находится в недеформированном состоянии, также в результате одноосного квазистатического растяжения наблюдаются как полосы локализованной деформации растяжения, так и сжатия. Переход от одной формы локализации к другой происходит в окрестности максимума напряжения и сопровождается резким уменьшением концентрационного параметра. Концентрационный параметр характеризует степень взаимодействия дефектов различных масштабных уровней посредством их упругих полей и может быть оценен по данным акустической эмиссии. Анализ зависимости пространственного периода полос локализованной деформации от размера расчетной ячейки показал, что полосы локализованной деформации имеют самоподобное строение и процесс деформирования локализован по границам зерен минералов, слагающих образец.
\end{abstract}

(c) Пантелеев Иван Алексеевич - кандидат физико-математических наук, научный сотрудник, e-mail: pia@icmm.ru Плехов Олег Анатольевич - доктор физико-математических наук, старший научный сотрудник, e-mail: роа@icmm.ru Наймарк Олег Борисович - доктор физико-математических наук, профеccop, e-mail: naimark@icmm.ru

Евсеев Антон Владимирович - кандидат технических наук, младший научный сотрудник, e-mail: evseev@mi-perm.ru Паньков Иван Леонидович - кандидат технических наук, старший научный сотрудник, e-mail: ivpan@mi-perm.ru Асанов Владимир Андреевич - доктор технических наук, e-mail: ava@mi-perm.ru

Ivan A. Panteleev - PhD in Physical and Mathematical Sciences, Researcher, e-mail: pia@icmm.ru Oleg A. Plekhov - Doctor of Physical and Mathematical Sciences, Senior Researcher,e-mail: poa@icmm.ru Oleg B. Naimark - Doctor of Physical and Mathematical Sciences, Professor, e-mail: naimark@icmm.ru Anton V. Evseev - PhD in Technical Sciences, Junior Researcher, e-mail: evseev@mi-perm.ru Ivan L. Pankov - PhD in Technical Sciences, Senior Researcher, e-mail: ivpan@mi-perm.ru Vladimir A. Asanov - Doctor of Technical Sciences, e-mail: ava@mi-perm.ru 


\title{
FEATURES OF STRAIN LOCALIZATION IN SYLVINITE UNDER TENSION
}

\author{
I.A. Panteleev' ${ }^{1}$, O.A. Plekhov ${ }^{1}$, O.B. Naimark ${ }^{1}$, A.V. Evseev², \\ I.L. Pankov², V.A. Asanov \\ ${ }^{1}$ Institute of Continuous Media Mechanics UB RAS, Perm, Russian Federation \\ ${ }^{2}$ Mining Institute UB RAS, Perm, Russian Federation
}

\author{
ARTICLE INFO \\ Received: 18 March 2015 \\ Accepted: 1 May 2015 \\ Published: 30 June 2015 \\ Keywords: \\ strain localization, \\ acoustic emission, \\ direct tension, \\ concentration parameter, \\ digital image correlation method
}

\begin{abstract}
This work is devoted to investigation of spatio-temporal localization of deformation at the direct uniaxial tensile of sylvinite with using of digital image correlation method and acoustic emission technique. For the implementation of direct tension there was used a special reversing device allowing to convert compressive force into tensile. Samples are concreted into the device before the experiment. Sequentially photographed images of side surfaces of specimens were used to reconstruct the distribution of components of displacement vector field and strain tensor on the side of sylvinite samples under uniaxial quasi-static tension. As result of obtained data analysis it is found that the deformation process occurs as two consistently following forms of spatiotemporal localization: a system of equidistantly located stationary foci of localized deformation and a single stationary dissipative localized structure. Areas of specimen outside the localization zones are in the undeformed state, also as result of uniaxial quasistatic tension of specimen it is established that the zones of localized deformation can be tensile as well as compressive. The transition from one localization form to another occurs in the maximum stress vicinity and is accompanied by a sharp decrease in the concentration parameter. Concentration parameter characterizes the degree of interaction between defects of different scale levels through their elastic fields and can be estimated from the acoustic emission data. Analysis of dependence of the spatial period of localized deformation bands on the size of computational cell showed that localized deformation bands are self-similar structures and process of deformation is localized at the grain boundaries minerals composing the sample.
\end{abstract}

\section{Введение}

В практике геомеханического обеспечения безопасного ведения горных работ одной из базовых задач является прояснение механизмов деформирования и разрушения горных пород при различных режимах и схемах нагружения. Актуальность данных исследований особенно остро ощущается при негативном проявлении геомеханических процессов, зачастую приводящих к катастрофическим последствиям. Проведение исследований, направленных на раскрытие механизмов деформирования и разрушения соляных пород, в конечном счете помогает принимать грамотные конструктивные и технологические решения, позволяющие минимизировать негативные последствия подобного рода. Особый интерес с точки зрения влияния на устойчивость краевых частей подработанного массива вызывают исследования механизмов деформирования при прямом растяжении. Данный вид испытаний, являясь технически наиболее сложным вследствие малых деформационных показателей, а также низкой контактной прочности соляных пород, обусловил широкое использование ряда косвенных методов (метод раскалывания, «бразильский» метод»), результаты которых трудно сопоставимы между собой и зачастую существенно различаются [1-3]. Современные технологические решения позволяют проводить прямые испытания образцов квазипластичных пород в условиях одноосного растяжения и исследовать особенности процесса деформирования с применением методов неразрушающего контроля. 
Ранее в работах $[4,5]$ было показано, что пластическое течение ряда горных пород при квазистатическом сжатии на всем протяжении деформирования вплоть до разрушения протекает локализовано. Формы макролокализации определяются законами деформационного упрочнения, действующими на соответствующих стадиях процесса. Полученные данные о формах макролокализации деформации при одноосном сжатии горных пород не отличаются от определенных ранее для щелочно-галоидных кристаллов [5]. На образцах сильвинита экспериментально удалось наблюдать только одиночную зону локализованной деформации, которая перемещается по поверхности образца со скоростью порядка $10^{-5} \mathrm{M} / \mathrm{c}$ (при скорости деформирования $610^{-5} \mathrm{c}^{-1}$ ). Такая одиночная деформационная волна соответствует стадии линейного деформационного упрочнения. Для других стадий деформационного течения при сжатии сильвинита регистрация форм локализации не реализуема из-за растрескивания образцов и отслоения породы с боковых граней.

Настоящая работа посвящена исследованию эволюции пространственно-временных форм макролокализации деформации при квазистатическом растяжении сильвинита на протяжении всего времени деформирования вплоть до образования магистральной трещины. Для восстановления полей компонент вектора перемещений и тензора деформации на боковой поверхности деформируемых образцов используется метод корреляции цифровых изображений. Этот метод является неразрушающим, неконтактным методом и широко применяется в настоящее время для исследования процессов локализации деформации и разрушения горных пород и бетонов [6-9]. Второй метод неразрушающего контроля, который был использован в настоящем исследовании, - метод акустической эмиссии. Этот метод позволяет проводить мониторинг процесса накопления повреждений в процессе нагружения $[10,11]$ и оценить отношение характерного размера дефектов к характерному расстоянию между ними.

\section{1. Условия проведения эксперимента}

Механические испытания проводились на призматических образцах сильвинита Верхнекамского калийного месторождения с поперечным размером $30 \times 30$ мм и высотой 90 мм. Характерный размер кристаллитов основных слагающих минералов галита и сильвина составлял порядка 0,5 мм. Образцы сильвинита испытывались на электромеханическом прессе с максимальным усилием 25 тонн. Для реализации прямого растяжения было использовано специальное реверсивное устройство, позволяющее преобразовывать усилие сжатия в усилие растяжение. Это устройство с подготовленным образцом показано на рис. 1. Эксперименты проводились при комнатной температуре. Для исключения погрешности, связанной с изгибом образца в реверсивном устройстве, измерение продольных (осевых) деформаций осуществлялось на поверхности образца с помощью двух датчиков консольного типа, располагаемых симметрично относительно центра образца. Скорость перемещения захватов составляла $1,6 \cdot 10^{-6} \mathrm{M} / \mathrm{c}\left(\sim 2 \cdot 10^{-5} \mathrm{c}^{-1}\right)$.

Для восстановления полей перемещения и деформации на поверхности деформируемых образцов сильвинита была использована цифровая оптическая система LaVision Strain Master. Алгоритм обработки потока видео данных основан на методе корреляции цифровых изображений (Digital Image Correlation - DIC). Этот метод позволяет с высокой точностью восстанавливать эволюцию перемещений и деформаций поверхности образцов из различных материалов при различных видах нагружения. Математический аппарат ме- 
тода DIC изложен, например, в [12-13]. Система Strain Master состоит из цифровой камеpы, LED подсветки, системы синхронизации и персонального компьютера со специализированным программным обеспечением. Отдельные элементы этой системы показаны на рис. 2. Цифровая камера имеет разрешение $1600 \times 1200$ пикселей и установлена на расстоянии порядка тридцати сантиметров от поверхности образца. Размер пикселя на регистрируемых изображениях составляет 7,4 $\mu$. Процесс деформирования образцов записывался камерой с частотой десять кадров в секунду. Поля перемещений и деформации рассчитывались для поверхностной области образца размером 54,5×29 мм.

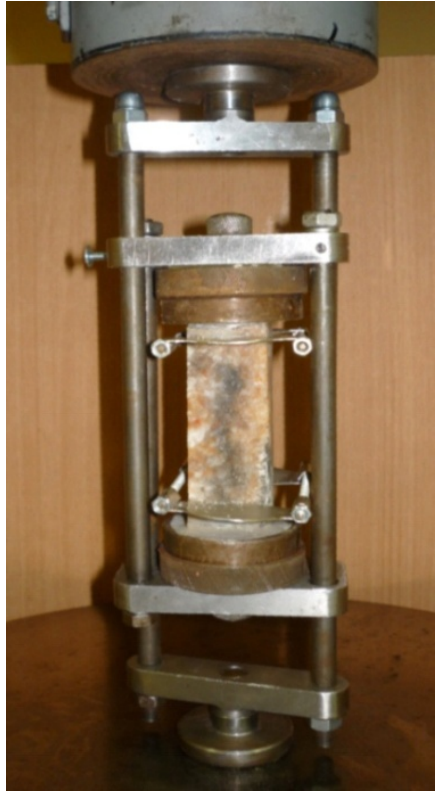

Рис. 1. Испытательное реверсивное устройство с подготовленным образцом

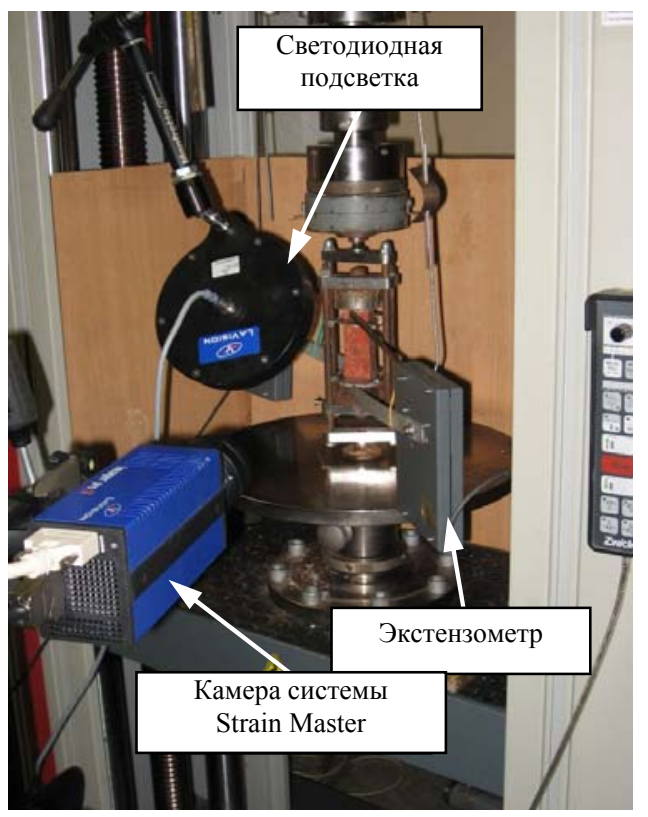

Рис. 2. Схема эксперимента на растяжение

Для исследования динамики развития повреждений в объеме образца был использован метод акустической эмиссии. Регистрация акустической эмиссии при квазистатическом растяжении образцов сильвинита осуществлялась с помощью восьмиканальной системы Amsy-5 (Vallen, Germany) с пьезокерамическими датчиками, установленными на поверхности образца. Частотный диапазон датчиков составляет от 350 до 2000 кГц.

Параметры акустической эмиссии, непрерывно регистрируемой в процессе нагружения образца, такие как скорость счета, распределение импульсов по энергии, суммарный счет, позволяют выявлять стадии деформирования с различной интенсивностью развития дефектов в объеме образца. Ранее в работах [14-18] для обнаружения перехода от стадии дисперсного накопления повреждений к их слиянию и образованию магистральной трещины был введен концентрационный параметр $K$. Этот параметр представляет собой отношение двух характерных размеров в материале с дефектами: характерного размера дефектов $L$ и среднего расстояния между ними $l$ :

$$
K=\frac{l}{L} .
$$

Концентрационный параметр характеризует степень взаимодействия дефектов различных масштабных уровней посредством упругих полей. В работах [14-15] показано, что когда концентрационный параметр достигает критической величины, равной 3, про- 
исходит объединение трещин с образованием магистрального разлома. Регистрируемые в процессе деформирования образцов события акустической эмиссии могут быть использованы для оценки изменения концентрационного параметра. Характерный размер дефектов можно определить, если принять предположение, что энергия одиночного импульса акустической эмиссии пропорциональна упругой энергии, которая выделилась при разгрузке шаровой области с радиусом $L / 2$ :

$$
E_{A E} \sim \frac{4}{3} \frac{\sigma^{2}}{E_{e}} \pi(L / 2)^{3},
$$

где $E_{e}$ - модуль Юнга; $\sigma$ - текущий уровень напряжения сжатия. Характерное расстояние между дефектами оценивается через объем образца $V$ и количество зарегистрированных импульсов $N$ как

$$
l \sim \sqrt[3]{\frac{V}{N}}
$$

Данные оценки хотя и не дают точных количественных значений, но позволяют качественно определить характер изменений концентрационного параметра в процессе деформирования образцов.

\section{2. Результаты экспериментов}

Характерные диаграмма нагружения и скорость акустической эмиссии для одноосного растяжения сильвинита представлены на рис. 3. Видно, что максимальная активность акустической эмиссии проявляется на активном участке деформирования в окрестности предельного напряжения. Еще одна характерная особенность - это небольшая величина предела прочности, около 1 МПа. Точками на кривой нагружения показаны моменты времени, для которых далее приводятся карты распределения поверхностной деформации.

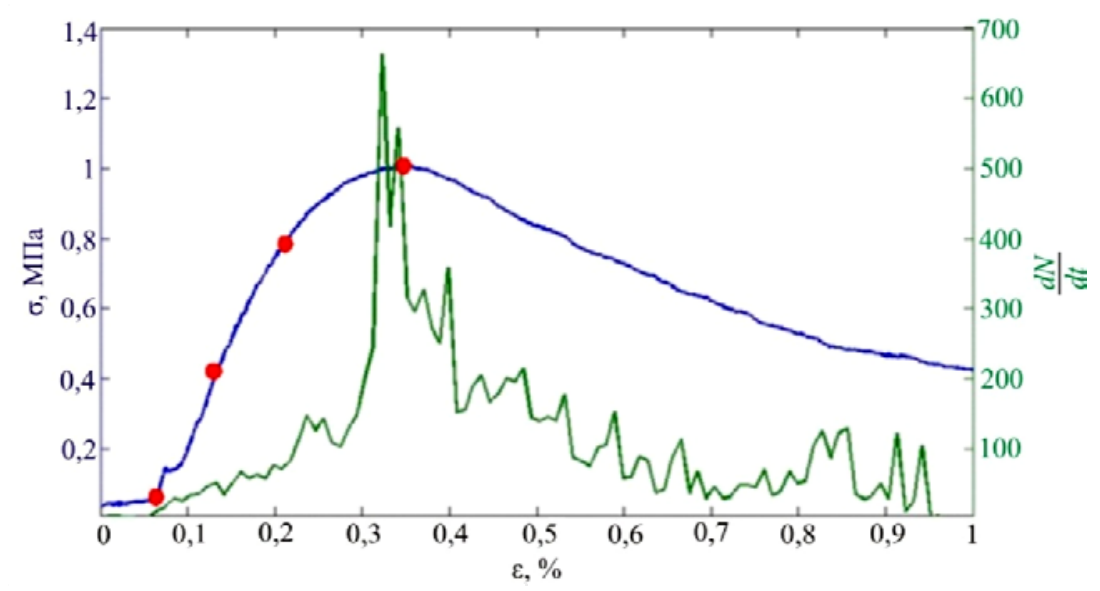

Рис. 3. Совмещенные диаграммы растяжения сильвинита и скорости поступления импульсов АЭ

Анализ эволюции распределения компонент тензора деформации на боковой поверхности образца сильвинита в процессе деформирования показал, что деформирование протекает неоднородно с самого начала нагружения. На рис. 4 представлены распределения 
продольной деформации на поверхности деформируемого образца сильвинита для различных моментов времени.

Неоднородность деформирования проявляется в виде системы эквидистантно расположенных зон локализованной деформации, ориентированных к оси нагружения под углом около $\pm \pi / 4$. Зоны локализованной деформации формируются с самого начала растяжения и с течением времени нагружения остаются неподвижными. Из распределения продольной деформации видно, что области материала вне зон локализации находятся в недеформированном состоянии.

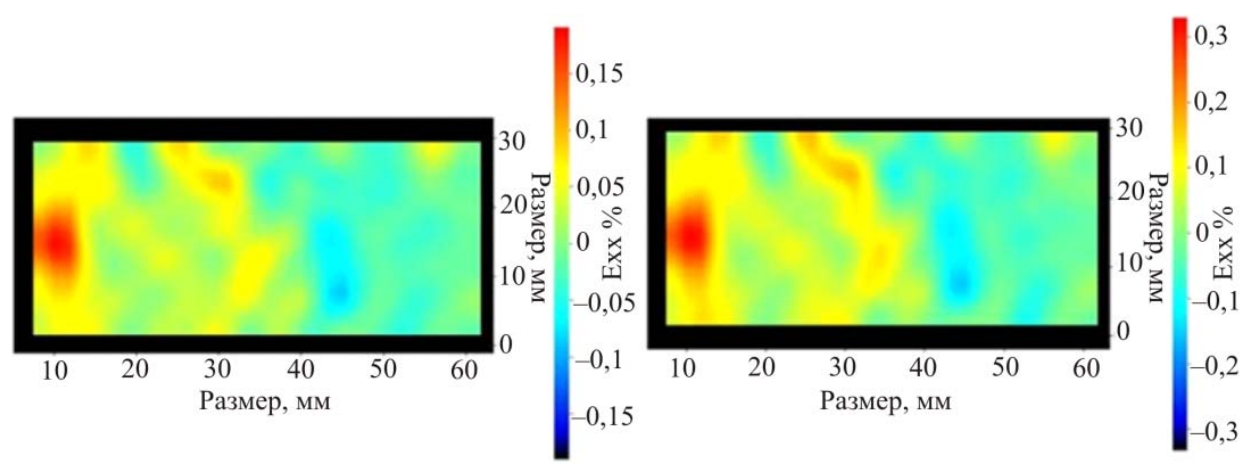

$a$

$\sigma$

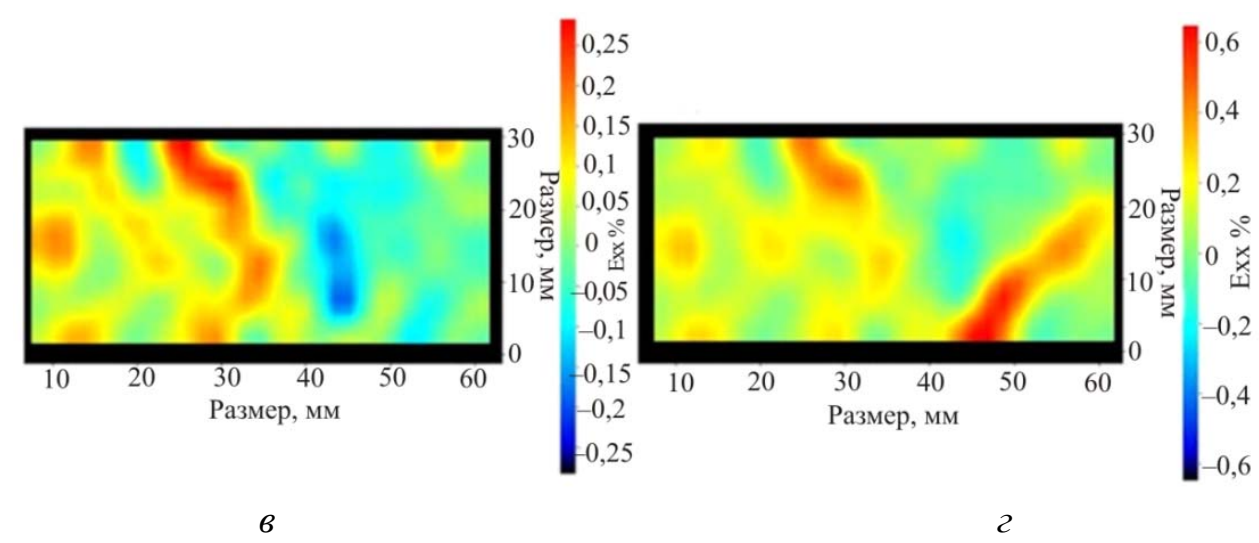

Рис. 4. Распределение продольной деформации на поверхности сильвинита при различном уровне общей деформации образца: $a-0,06 \% ; \sigma-0,12 \% ; в-0,21 \% ; 2-0,36 \%$

Внутри зон локализации деформация достигает уровня 0,5 \% при общей деформации образца 0,36 \%. Распределение поперечной деформации на поверхности сильвинита при растяжении является также неоднородным, хотя система эквидистантно расположенных зон просматривается не так четко.

Периодическое расположение зон локализованной деформации более явно видно на зависимости продольной деформации от времени для средней линии образца. Эта зависимость представлена на рис. 5. Как видно из рисунка, в локализованных зонах деформация может быть как деформацией растяжения, так и деформацией сжатия. Этот факт является нетривиальным с учетом того, что на макромасштабе к образцу приложено растягивающее усилие. Пространственный период расположения зон локализованной деформации составляет 13,6 мм. Из анализа расположения максимумов деформации в течение нагружения была получено, что деформация в зонах локализации непрерывно растет, а максимум деформации перемещается от зоны к зоне. Процесс деформирования 
в виде эквидистантно расположенных локализованных зон протекает вплоть до момента, когда напряжение в образце достигает своего максимума. В этот момент происходит смена формы макролокализации: процесс деформирования продолжается только в одной из зон локализации и заканчивается разрушением, тогда как в других зонах деформирование полностью останавливается (см. рис. 5).

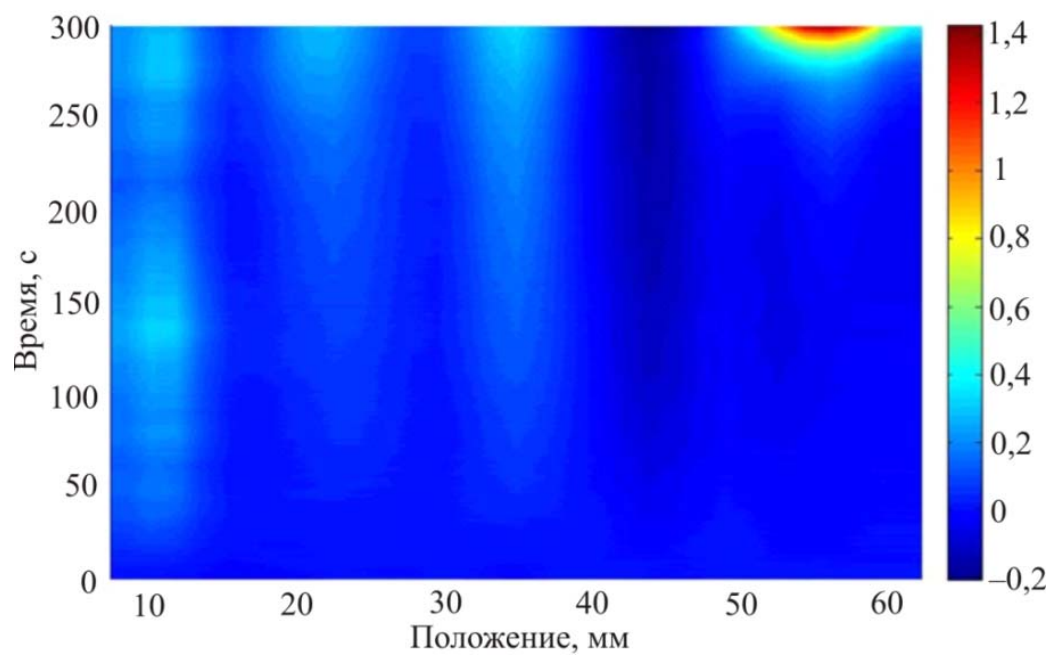

Рис. 5. Эволюция продольной деформации для средней линии образца

Таким образом, следуя терминологии, введенной в работе [19], при квазистатическом растяжении образцов сильвинита процесс деформирования реализуется в виде двух последовательных форм пространственно-временной локализации: системы эквидистантно расположенных стационарных очагов локализованной деформации и одиночной стационарной диссипативной локализованной структуры, деформация внутри которой растет лавинообразно и заканчивается макроразрушением.

В отличие от ранее полученных результатов для ряда горных пород, металлов и сплавов $[5,19]$ реализация каждой из форм макролокализации при растяжении сильвинита не определяется соответствующей стадией кривой пластического течения (линейного деформационного упрочнения, параболического деформационного упрочнения, стадии предразрушения), а зависит от уровня приложенного напряжения.

При использовании метода корреляции цифровых изображений для восстановления поля перемещений и компонент тензора деформации поверхность образца в каждом кадре покрывается однородной сеткой с размером ячейки $L \times L$. Далее для определения направления и величины смещения в каждой ячейке вычисляется положение и величина максимума кросс-корреляционной функции яркости в этой ячейке для двух соседних кадров. Размер ячеек при разбиении задается произвольно и ограничен снизу размером $6 \times 6$ пикселей, связанным с ограничением схемы вычисления кросс-корреляционной функции. Выбор размера ячейки совпадает с выбором пространственного масштаба наблюдения, определяющего необходимую степень детализации исследуемого процесса изменения напряженно-деформируемого состояния. Уменьшение размеров ячейки при построении зависимости продольной деформации от времени для средней линии образца позволило установить, что каждая из зон локализованной деформации состоит из эквидистантно расположенных зон меньшего размера, которые в свою очередь состоят из полос еще меньшего масштаба. Этот результат указывает на самоподобное строение зон локализа- 
ции деформации. Полученная зависимость пространственного периода $T_{l o c}$ расположения очагов локализованной деформации от размера ячейки разбиения имеет линейный вид (рис. 6):

$$
T_{l o c}=3,8 L+0,49 .
$$

В предельном случае при стремлении размера ячейки к нулю, что соответствует масштабу наблюдения, соизмеримому с межатомным расстоянием, пространственный период расположения зон стремится к величине 0,5 мм, совпадающей с характерным размером щелочно-галоидных кристаллитов слагающих сильвинит.

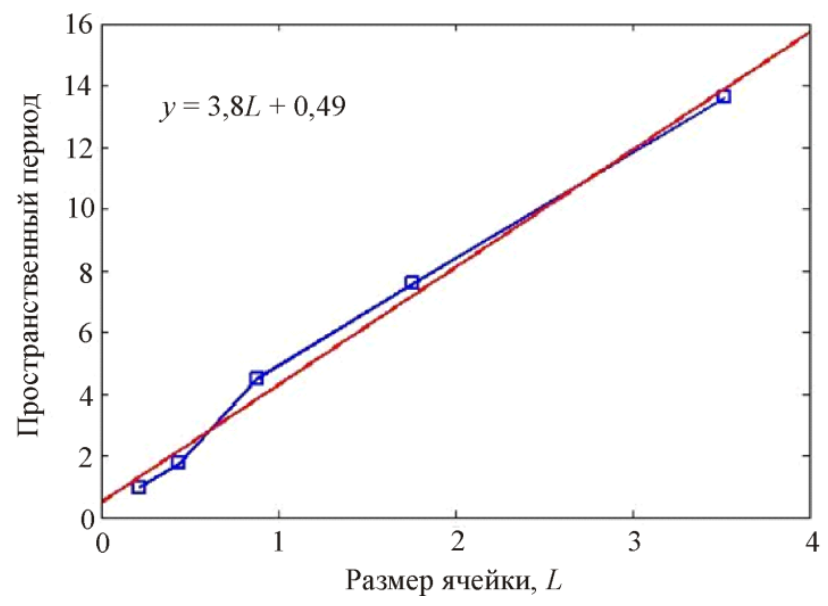

Рис. 6. Зависимость пространственного периода локализованных структур от размера ячейки разбиения (сплошная линия - аппроксимация)

Это означает, что с самого начала растяжения процесс деформирования протекает преимущественно по границам кристаллитов. Неизменность формы макролокализации деформации на стадии активного деформирования указывает на то, что дислокационное течение внутри кристаллитов не реализуется и связано это с очень низкой когезионной прочностью их границ. Этот факт объясняет выявленное противоречие с закономерностями эволюции пространственно-временных форм макролокализации деформации, обнаруженными при деформировании ряда горных пород, металлов и монокристаллов $[4,5]$.

Для сравнения с обнаруженными формами локализации деформации была построена эволюция концентрационного параметра в процессе деформирования сильвинита. Эти зависимость представлена на рис. 7. Из рисунка видно, что начиная с 100-й секунды, когда количество зарегистрированных импульсов акустической эмиссии становится существенным, величина концентрационного параметра слабо изменяется. Однако в окрестности максимума напряжений происходит резкое уменьшение этого параметра. Скачок концентрационного параметра соответствует резкому падению напряжения и связан с появлением магистральной трещины в образце, который продолжает сопротивляться приложенной нагрузке. Таким образом, постоянное значение концентрационного параметра соответствует деформированию образца в форме эквидистантных локализованных зон, а резкое изменение этого параметра соответствует переходу от одной формы локализации к другой.

Проведенные экспериментальные исследования позволили подтвердить теоретические предположения о реализации пространственно локализованного деформирования 
материалов с дефектами в виде двух последовательно следующих форм (периодических стационарных очагов и одиночной диссипативной структуры). Эти предположения впервые были высказаны в работе [20] и получили свое развитие в работах $[17,18,21]$, посвященных развитию статистико-термодинамической модели поведения среды с дефектами в поле приложенных напряжений. С использованием современного экспериментального оборудования впервые показано самоподобное строение зон локализации деформации при квазистатическом растяжении сильвинита.

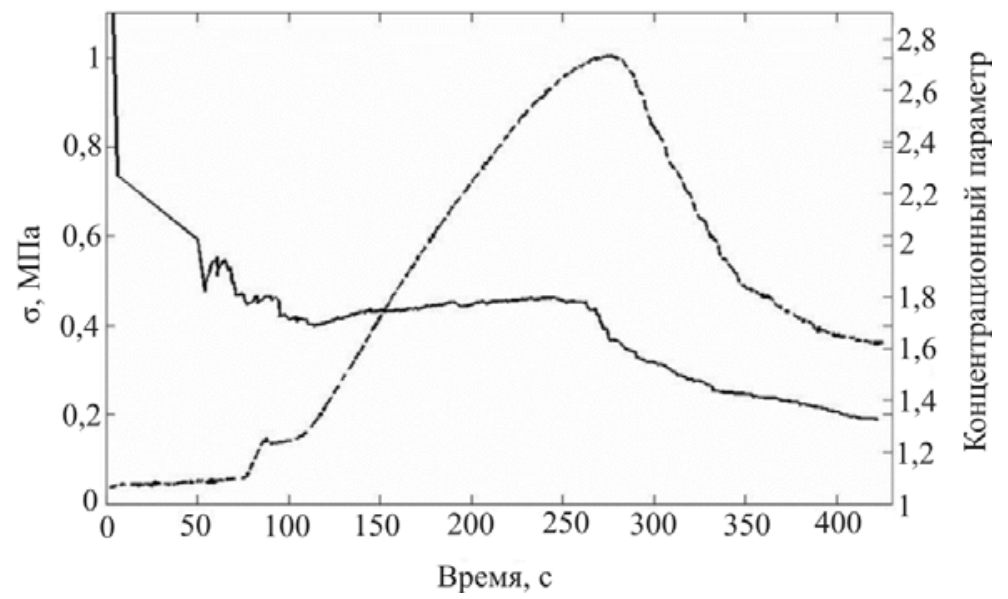

Рис. 7. Совмещенные зависимости напряжения (пунктирная линия) и концентрационного параметра (сплошная линия) от времени деформирования

\section{Выводы}

Для исследования процессов пространственно-временной локализации деформации при одноосном квазистатическом растяжении сильвинита был использован метод корреляции цифровых изображений. Данные акустической эмиссии, регистрируемой при накоплении повреждений в течение нагружения образца, позволили оценить изменения концентрационного параметра. Было показано, что процесс деформирования локализован в пространстве в течение всего времени нагружения и реализуется в виде двух последовательно следующих форм пространственно-временной локализации: системы эквидистантно расположенных стационарных очагов локализованной деформации и одиночной стационарной диссипативной локализованной структуры. Пространственный период локализованных зон составляет порядка 13,6 мм. Переход от одной формы к другой происходит в окрестности максимума напряжения и сопровождается резким уменьшением концентрационного параметра, что указывает на появление магистральной трещины в объеме образца.

Работа выполнена при поддержке Российского научного фонда (грант 14-19-01173). 


\section{Библиографический список}

1. Butenuth C. Comparison of tensile strength values of rocks determined by point load and direct tension tests // Rock Mech. Rock Engng. - 1997. - Vol. 30. - No. 1. - P. 65-72. DOI: 10.1007/BF01020114

2. Efimov V.P. The rock strength in different tension conditions // Journal of Mining Science. 2009. - Vol. 45. - No. 6. - P. 569-575.

3. Erarslan N., Williams D.J. Experimental, numerical and analytical studies on tensile strength of rocks // International Journal of Rock Mechanics \& Mining Sciences. - 2012. - Vol. 49. - P. 21-30.

4. О неоднородности деформации при сжатии сильвинита / С.А. Баранникова, М.В. Надежкин, Л.Б. Зуев, В.М. Жигалкин // ПЖТФ. - 2010. - Т. 36, № 11. - С. 38-45.

5. Баранникова С.А., Надежкин М.В., Зуев Л.Б. О локализации пластического течения при сжатии кристаллов $\mathrm{NaCl}$ и $\mathrm{KCl} / /$ ФТТ. - 2009. - Т. 51, № 6. - С. 1081-1086.

6. Choi S., Shah SP. Measurement of deformation on concrete subject to compression using image correlation // Exp. Mech. - 1997. - Vol. 37. - No. 3. - P. 307-313. DOI: 10.1007/BF02317423

7. Lawler J.S., Kean D.T., Shah S.P. Measuring three-dimensional damage in concrete under compression // ACI Mater J. - 2001. - Vol. 98. - No. 6. - P. 465-475.

8. Bhandari A.R., Inoue J. Strain localization in soft rock - a typical rate-dependent solid: experimental and numerical studies // Int. J. Numer. Anal. Meth. Geomech. - 2005. - Vol. 29. - P. 1087-1107. DOI: $10.1002 /$ nag.446

9. Experimental study on damage evolution of rock under uniform and concentrated loading conditions using digital image correlation / H. Song, H. Zhang, D. Fu, Y. Kang, G. Huang, C. Qu, Z. Cai // Fatigue Fract. Engng. Mater. Struct. - 2013. - Vol. 36. - No. 8. - P. 760-768. DOI: 10.1111/ffe.12043

10. Source analysis of acoustic emission in Aue granite cores under symmetric and asymmetric compressive loads / A. Zang, F.C. Wagner, S. Stanchits, G. Dresen, R. Andresen, M.A. Haidekker // Geophys. J. Int. - 1998. - Vol. 135. - P. 1113-1130.

11. Zang A., Wagner F.C., Dresen G. Acoustic emission, microstructure and damage model of dry and wet sandstone stressed to failure // J. Geophys. Res. - 1996. - Vol. 101. - P. 17507-17521.

12. Determination of displacements using an improved digital correlation method / M.A. Sutton, W.J. Wolters, W.H. Peters, W.F. Ranson, S.R. McNeil // Image and Vision Computing. - 1983. - Vol. 1. No. 3. - P. 133-139.

13. Sutton M.A., Orteu J.J., Schreier H.W. Image Correlation for Shape, Motion and Deformation Measurements: Basic Concepts, Theory and Applications. - Springer, 2009. -316 p.

14. A Two-stage Model of Fracture of Rocks / V. Kuksenko, N. Tomilin, E. Damaskinskaya, O. Lockner // PAGEOPH. - 1996. - Vol. 146. - No. 2. - P. 253-263. DOI: 10.1007/BF00876492

15. Куксенко В.С. Диагностика и прогнозирование разрушения крупномасштабных объектов // ФТТ. - 2005. - Т. 47, № 5. - С. 788-792.

16. Плехов О.А., Пантелеев И.А. Оптимизация предсказания времени разрушения твердых тел на основе представления об иерархической природе деформации и анализа истории нагружения // Физ. мех. - 2008. - Т. 11, № 6. - С. 53-59.

17. Пантелеев И.А, Плехов О.А., Наймарк О.Б. Нелинейная динамика структур обострения в ансамблях дефектов как механизм формирования очагов землетрясения // Физика Земли. 2012. - № 6. - С. 43-55.

18. Пантелеев И.А., Плехов О.А., Наймарк О.Б. Модель геосреды с дефектами: коллективные эффекты развития несплошностей при формировании потенциальных очагов землетрясений // Геодинамика \& Тектонофизика. - 2013. - Т. 4, № 1. - С. 37-51. DOI:10.5800/GT2013410090

19. Autowave model of localized plastic flow of solids / L.B. Zuev, V.I. Danilov, S.A. Barannikova, V.V. Gorbatenko // Physics of Wave Phenomena. - 2009. - Vol. 17. - No. 1. - P. 66-75. DOI: $10.3103 / \mathrm{S} 1541308 \mathrm{X} 09010117$ 
20. Наймарк О.Б. О деформационных свойствах и кинетике разрушения твердых тел с микротрещинами. О термодинамике деформирования и разрушения твердых тел с микротрещинами. Свердловск, 1982. - С. 3-34.

21. Structural-scaling transitions and thermodynamic and kinetic effects in submicro-(nano-)crystalline bulk materials / O.B. Naimark, Yu.V. Bayandin, V.A. Leontiev, I.A. Panteleev, O.A. Plekhov // Physical Mesomechanics. - 2009. - Vol. 12. - No. 5-6. - P. 239-248.

\section{References}

1. Butenuth C. Comparison of tensile strength values of rocks determined by point load and direct tension tests. Rock Mech. Rock Engng., 1997, vol. 30, no. 1, pp. 65-72. DOI: 10.1007/BF01020114

2. Efimov V.P. The rock strength in different tension conditions. Journal of Mining Science, 2009, vol. 45 , no. 6 , pp. 569-575.

3. Erarslan N., Williams D.J. Experimental, numerical and analytical studies on tensile strength of rocks. International Journal of Rock Mechanics \& Mining Sciences, 2012, vol. 49, pp. 21-30.

4. Barannikova S.A., Nadezhkin M.V., Zuev L.B., Zhigalkin V.M. On Inhomogeneous Straining in Compressed Sylvinite. Technical Physics Letters, 2010, vol. 36, no. 6, pp. 507-510. DOI: 10.1134/S1063785010060064

5. Barannikova S.A., Nadezhkin M.V., Zuev L.B. On the Localization of Plastic Flow under Compression of $\mathrm{NaCl}$ and $\mathrm{KCl}$ Crystals. Physics of the Solid State, 2009, vol. 51, no. 6, pp. 1142-1148. DOI: 10.1134/S1063783409060109

6. Choi S., Shah SP. Measurement of deformation on concrete subject to compression using image correlation. Exp. Mech., 1997, vol. 37, no. 3, pp. 307-13. DOI: 10.1007/BF02317423

7. Lawler J.S., Kean D.T., Shah S.P. Measuring three-dimensional damage in concrete under compression. ACI Mater J., 2001, vol. 98, no. 6, pp. 465-475.

8. Bhandari A.R., Inoue J. Strain localization in soft rock - a typical rate-dependent solid: experimental and numerical studies. Int. J. Numer. Anal. Meth. Geomech., 2005, vol. 29, pp. 1087-1107. DOI: 10.1002/nag. 446

9. Song H., Zhang H., Fu D., Kang Y., Huang G., Qu C., Cai Z. Experimental study on damage evolution of rock under uniform and concentrated loading conditions using digital image correlation. $\mathrm{Fa}$ tigue Fract. Engng. Mater. Struct., 2013, vol. 36, no. 8, pp. 760-768. DOI: 10.1111/ffe.12043

10. Zang A., Wagner F.C., Stanchits S., Dresen G., Andresen R., Haidekker M.A. Source analysis of acoustic emission in Aue granite cores under symmetric and asymmetric compressive loads. Geophys. J. Int., 1998, vol. 135, pp. 1113-1130.

11. Zang A., Wagner F.C., Dresen G. Acoustic emission, microstructure and damage model of dry and wet sandstone stressed to failure. J. Geophys. Res., 1996, vol. 101, pp. 17507-17521.

12. Sutton M.A., Wolters W.J., Peters W.H., Ranson W.F., McNeil S.R. Determination of displacements using an improved digital correlation method. Image and Vision Computing, 1983, vol. 1, no. 3, pp. 133-139.

13. Sutton M.A., Orteu J.J., Schreier H.W. Image Correlation for Shape, Motion and Deformation Measurements: Basic Concepts, Theory and Applications. Springer, 2009. 316 p.

14. Kuksenko V., Tomilin N., Damaskinskaya E., Lockner O. A Two-stage Model of Fracture of Rocks. PAGEOPH, 1996, vol. 146, no. 2, pp. 253-263. DOI: 10.1007/BF00876492

15. Kuksenko V. Diagnostics and Forecasting of Breakage of Large-Scale Objects. Physics of the Solid State, 2005, vol. 47, no. 5, pp. 812-816.

16. Plekhov O.A., Panteleev I.A. Optimization of fracture time prediction for solids using the concept of deformation hierarchy and loading history analysis. Physical Mesomechanics, 2009, vol. 12, no. 1-2, pp. 60-65. DOI:10.1016/j.physme.2009.03.007 
17. Panteleev I.A., Plekhov O.A., Naimark O.B. Nonlinear dynamics of the blow-up structures in the ensembles of defects as a mechanism of formation of earthquake sources. Izvestiya. Physics of the Solid Earth, 2012, vol. 48, no. 6, pp. 504-515. DOI: 10.1134/S1069351312060055

18. Panteleev I.A., Plekhov O.A., Naimark O.B. Model' geosredy s defektami: kollektivnye effekty razvitiia nesploshnostei pri formirovanii potentsial'nykh ochagov zemletriasenii [Model of geomedia containing defects: collective effects of defects evolution during formation of potential earthquake foci]. Geodynamics \& Tectonophysics, 2013, vol. 4, no. 1, pp. 37-51. DOI:10.5800/GT2013410090

19. Zuev L.B., Danilov V.I., Barannikova S.A., Gorbatenko V.V. Autowave model of localized plastic flow of solids. Physics of Wave Phenomena, 2009, vol. 17, no. 1, pp. 66-75. DOI: 10.3103/S1541308X09010117

20. Naimark O.B. O deformatsionnykh svoistvakh i kinetike razrusheniia tverdykh tel $\mathrm{s}$ mikrotreshchinami [About deformation properties and kinetics of damage of solid with microcracks]. Preprint. Sverdlovsk. 1982. pp. 3-34.

21. Naimark O.B., Bayandin Yu.V., Leontiev V.A., Panteleev I.A., Plekhov O.A. Structural-scaling transitions and thermodynamic and kinetic effects in submicro-(nano-)crystalline bulk materials. Physical Mesomechanics, 2009, vol. 12, no. 5-6, pp. 239-248. 